\title{
Ellipse ou illocutoire? Quelques aspects gestuels et prosodiques dans le discours en L2
}

Ellipsis or Illocution? Some Gestural and Prosodic Aspects of Second Language

(L2) Speech

Tsuyoshi Kida et Martine Faraco

\section{(2) OpenEdition}

\section{Journals}

Édition électronique

URL : https://journals.openedition.org/tipa/741

DOI : 10.4000/tipa.741

ISSN : 2264-7082

Éditeur

Laboratoire Parole et Langage

Référence électronique

Tsuyoshi Kida et Martine Faraco, «Ellipse ou illocutoire? Quelques aspects gestuels et prosodiques dans le discours en L2 ", TIPA. Travaux interdisciplinaires sur la parole et le langage [En ligne], 29 | 2013, mis en ligne le 17 décembre 2013, consulté le 10 mars 2023. URL : http://journals.openedition.org/ tipa/741 ; DOI : https://doi.org/10.4000/tipa.741

Ce document a été généré automatiquement le 10 mars 2023

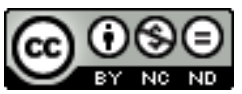

Creative Commons - Attribution - Pas d'Utilisation Commerciale - Pas de Modification 4.0 International - CC BY-NC-ND 4.0

https://creativecommons.org/licenses/by-nc-nd/4.0/ 


\section{Ellipse ou illocutoire? Quelques aspects gestuels et prosodiques dans le discours en $\mathrm{L} 2$}

Ellipsis or Illocution? Some Gestural and Prosodic Aspects of Second Language (L2) Speech

Tsuyoshi Kida et Martine Faraco

\section{Introduction}

1 Cette réflexion sur la compréhension orale en langue seconde (L2) dans une interaction exolingue porte sur le « totexte » ou énoncé total, défini par Cosnier (2012:104) comme l'ensemble formé du texte (verbal) et du cotexte. En effet, dans une interaction en face à face, la structure du discours oral est jalonnée par un certain nombre d'indices gestuels et prosodiques aussi bien en situation de communication endolingue qu'exolingue (Kida, à paraître; Kida \& Faraco, 2009). Or, la mise en emphase (accent gestuel) obéit à des contraintes propres à chaque langue (voir, entre autres, les travaux de Müller, 1994, Kita, 1993, Stam, 2006), ce qui conduit les locuteurs à utiliser une sorte de syntaxe gestuelle régie par leur langue dominante. C'est ainsi que ce type d'« accent gestuel » (Creider, 1978, 1986 ; Kida \& Faraco, 2009) ou « manual accent » (Kellerman \& van Hoof, 2003) est souvent transféré d'une langue à l'autre, lors de l'apprentissage d'une langue seconde (Stam, 1998 ; van Hoof \& Kellerman 2001 ; Yoshioka \& Kellerman, 2006).

2 À partir de ces constats, il semble cohérent de conduire une étude sur les gestes produits par des apprenants en L2 puisque le transfert du comportement d'accent manuel de la langue maternelle à la L2 prouve l'existence d'une grammaticalité de la modalité gestuelle dans le discours. Dans une précédente étude (Kida et Faraco, à paraître), nous avons travaillé sur l'appropriation de la mise en gestes de japonophones apprenant le français comme L2, donc pour deux langues ayant une 'grammaticalité' gestuelle comparable, tout au moins pour ce qui est de l'accent manuel. En effet, 
japonais et français sont tous deux classés dans le groupe des langues à cadrage verbal et non dans celui des langues à cadrage satellite (Talmy, 1991 et 2000, entre autres).

3 Nos observations précédentes portant sur l'appropriation de l'oral, du prosodique et du gestuel d'une langue cible (le français) par des locuteurs non natifs (LNNs), cette contribution se propose de préciser ces observations. Après quelques remarques générales sur la façon dont se comportent les modalités gestuelle et vocale en cas d'absence du verbal dans le discours explicatif d'un locuteur natif (LN) qui tente de se faire comprendre de son partenaire non natif, nous verrons comment la focalisation non verbale fonctionne au plan pragmatique en cas d'ellipse verbale chez le LNN.

\section{Préambule}

L'ellipse est une figure de rhétorique qui consiste à omettre un ou plusieurs éléments du discours représentant un fait sans compromettre la compréhension de celle ou celui à qui ce discours est destiné. Cela présuppose que l'actualisation des éléments omis, mais potentiellement présents dans la situation du discours, reviendrait à une « mise à jour » qui risque parfois d'inclure un effet particulier et de donner un autre sens que le sens d'origine. Ainsi, prenons le cas où un enfant se montre réticent à commencer à manger son plat. Si sa mère dit mange ce plat, au lieu de l'expression elliptique mange, l'enfant, s'il y a un choix de plats sur la table, peut tout aussi bien penser : «je dois manger ce plat, sans être obligé de manger les autres ", ou bien : " après notre dispute, elle m'a préparé mon plat préféré et, pour faire la paix, elle attire mon attention sur ce plat ». On y décèle un principe d'économie - puisque le discours elliptique suffit pour transmettre l'intention - mais aussi un principe de "sécurité », consistant à éviter un éventuel malentendu. En tout cas, l'ellipse au sens premier présuppose que les éléments omis sont compris ou mutuellement partagés.

5 Le Portail linguistique du Canada donne des indications sur la fonction habituelle d'économie de mots qu'autorise l'ellipse pour ne garder que les mots les plus importants, c'est-à-dire indispensables à sa compréhension. L'énoncé se fait donc plus dynamique, plus condensé en sens, d'où l'efficacité de l'ellipse dans les textes publicitaires ou journalistiques. Précisément, cette figure de style donne concrètement la possibilité d'accélérer le débit et de rapprocher les mots chargés de sens, suivant la logique du 間 (ma japonais) - ce vide qui unit, cet espace qui relie - cher à Barthes (1970) $)^{1}$.

6 Dans une interaction avec un LNN, la présupposition sur laquelle l'ellipse s'appuie s'avère être quelque peu différente. Dans le foreigner talk (Ferguson, 1977), il s'agit de sortes d'ajustements grammaticaux de la part d'un LN, lesquels peuvent impliquer la suppression de la copule, de l'article, de la flexion, du pronom sujet (Larsen-Freeman \& Long, 1991 ; Long, 1996), ces éléments omis étant présupposés être des obstacles qui entravent la compréhension du LNN. Autrement dit, le LN omet un ou des éléments du discours pour augmenter la compréhensibilité de son énoncé par un LNN.

7 Le Trésor de la langue Française Informatisé (TLFI) retient un tel caractère ambivalent de l'ellipse : nous trouvons : « Ling. Omission d'un ou plusieurs mots dans un énoncé dont le sens reste clair » puis, un peu plus loin : « Péj. Omission d'un ou de plusieurs énoncés qui rend le sens difficile à comprendre. » C'est donc dans l'accès au sens par 
l'interlocuteur de ce que veut dire le locuteur que l'ellipse, en général, trouve toute sa contradiction, car elle peut tout aussi bien faciliter la compréhension que la brouiller.

De là, on peut imaginer qu'il y a relation entre ellipse et niveau linguistique du LNN avec qui le LN interagit. Pour asseoir la réflexion sur des éléments concrets, citons quelques exemples d'ellipses, tirés du corpus Kida (2005). Il est vrai que les différents types de focalisation par ellipse qui suivent sont extraits de la situation où les interlocuteurs ne se voient pas, mais nous les trouvons aussi en face à face, quand les interactants se voient :

Pronom avec référence au geste :

- on fait comme ça

- on fait ça

Article défini (anaphore) :

- (aubergine) tu peux laisser/on enlève la peau (de ø)

Ellipse courante :

- on mange (ø) avec des pains grillés

Verbes typiques de la cuisine (ellipse de genre discursif):

- on sort (ø) du feu

- on fait (ø) à la vapeur

- pour mettre au four $(\varnothing)$

- on laisse (ø) au four/au chaud/tranquille

- on met (ø) dans un bol en bois

- on fait revenir (ø) dans une poêle

- on fait bouillir (ø) dans une grande casserole

- tu laisses cuire (ø) comme un gratin/longtemps/un petit peu

- tu mélanges (ø)

- on ajoute (ø) dans la purée

Emphase-répétition :

- j'écrase-j'écrase-j'écrase (ø) complètement

- on écrase (ø) bien-bien-bien

Métaphore :

- j'ouvre les (ø de) haricots et je prends des petits (ø de) haricots

- je mets cette préparation dans les haricots ( $\varnothing$, métonymie)

Expansion :

- (les feuilles de basilic) et j'ajoute (ø) dans le bol avec l'ail, l'huile d'olive, la tomate et le basilic

Contraction:

- tu coupes les petits morceaux de poisson (pour dire « le poisson en morceaux»)

NB. () est une information contextuelle, qui n'est pas forcément l'antécédent immédiat.

En examinant les occurrences de l'énoncé elliptique du LN en interaction avec sept LNNs dans une situation où les deux interlocuteurs se voient et une autre où ils sont séparés par un écran opaque, Kida (2008) relève certaines tendances : 1 ) le nombre des ellipses du LN augmente quand les partenaires non natifs sont de niveaux linguistiques moins avancés, mais la condition de visibilité/non-visibilité n'offre pas de grand contraste; 2) au niveau avancé, par contre, l'ellipse du LN augmente en situation d'écran par rapport à la situation de face à face. Globalement, l'ellipse peut être une stratégie d'interaction du LN pour que le discours soit plus aisé et rapide à suivre. Soulignons toutefois que l'utilisation de l'ellipse est non seulement liée au niveau de 
compétence discursive de l'interlocuteur non natif, mais aussi à la disponibilité de l'information visuelle qui accompagne le discours.

11 On peut alors supposer que le rapport entre focalisation discursive, ellipse et indice visuel demeure plus complexe en situation de face à face qu'en privation visuelle. Si, par exemple, les interactants ne disposaient pas du biais visuel, ils seraient privés d'une mise en focalisation subtile et devraient, de ce fait, modifier leur mode de communication. Dans notre corpus, le LN semble abuser non seulement de l'emphase intonative, mais aussi de l'ellipse en situation de privation visuelle pour tenter de faciliter la compréhension du partenaire. Ainsi, Kida (2003 : 285) relève, comme nous venons de le voir, divers types d'ellipse attestés en privation visuelle (voir Kida, 2003 : 285) permettant de dresser une typologie des éléments omissibles du discours du LN en interaction avec le LNN. Il est à noter que le comportement est similaire au moment où l'intercompréhension est en danger en face à face. Cela veut dire que le LN use d'un moyen tantôt intonatif tantôt verbal tantôt les deux au moment de la focalisation discursive comme stratégie d'interaction face aux LNNs.

Par ailleurs, il ressort de nos observations que le niveau de compétence discursive du LNN influence la tonalité prosodique de l'échange et cela est très nettement perceptible en privation visuelle. En effet, seule l'emphase intonative peut être utilisée dans une négociation de sens puisque les indices visuels permettant d'aider à la compréhension font défaut en privation visuelle. Ainsi, un LNN avancé se montre-t-il très réceptif et, par moments, l'objet focalisé est alors négocié par les interactants sur la base du partage de la compréhension fonctionnelle des ellipses. Par contre, d'autres sujets moins avancés se contentent d'adopter une "stratégie d'écoute " en acquiesçant automatiquement aux différentes emphases du LN. En conséquence, l'intention du LN visant à assurer une intercompréhension à l'aide de l'ellipse et de la focalisation est souvent vouée à l'échec avec les LNNs moins avancés.

L'omission d'un ou plusieurs éléments est également courante dans le discours des LNNs en L2, mais sans doute celle-ci provient-elle d'une compétence discursive à la fois déficitaire et instable par rapport aux normes de la langue cible. En effet, chez les LNNs qui tentent d'assurer l'intercompréhension, s'observe un comportement spécifique consistant à résoudre le problème de communication de façon stratégique et la résolution par voie gestuelle est observée au nombre de ces stratégies (Gullberg, 1998). Or, l'ellipse du discours des LNNs n'est pas seulement d'ordre stratégique, mais doit remplir aussi une fonction rhétorique et pragmatique (Kida \& Faraco, 2003), au même titre que certains éléments prosodiques du discours. Il conviendrait alors d'examiner l'ellipse avec les éléments gestuels et prosodiques du discours, pour appréhender les rôles que jouent ces éléments en l'absence d'information verbale.

\section{Cadre d'analyse}

14 Ce travail porte sur des séquences filmées de conversations en français d'interlocuteurs procédant mutuellement à l'explication d'une recette de cuisine (Kida, à paraître ${ }^{2}$ ). Les LNNs sont, pour la plupart, des japonophones (de niveau linguistique variable ${ }^{3}$ ), mais également des locuteurs d'autres langues maternelles (danois, espagnol, anglais). Les enregistrements ont eu lieu dans une chambre anechoïque de l'université, les participants ont interagi en dyade, sur le schéma LN-LNN ou LNN-LNN dans le cadre d'une conversation thématique pour apprendre une recette au partenaire (Ra) et pour 
apprendre du partenaire une recette $(\mathrm{Rb})$ : en face à face (R1) et séparés par un écran en situation de privation visuelle (R2). Il y a aussi une partie de l'enregistrement consacrée à une conversation libre (Conv.). L'observation du présent travail s'appuie sur les enregistrements d'interactions LN/LNN et LNN/LNN, ce dernier cas impliquant au moins un LNN japonophone. Dans ce cadre, nous nous intéresserons aux échanges contenant des exemples d'ellipse verbale, fait caractéristique de l'oral et a fortiori de l'oral en L2.

\section{Déictique et idéographique}

ce qui suit, nous verrons que la compréhension discursive repose aussi sur des gestes tels qu'idéographiques ou déictiques. Efron (1972: 96-98) explicite la différence entre physiographique et idéographique selon sa terminologie ${ }^{4}$ (ce dernier étant généralement désigné comme discursif) de la façon suivante : le geste idéographique se caractérise comme étant celui qui "traces or sketches out in the air the "paths" and "directions" of the thought-pattern ", qu'il appelle " course of the ideational process " (p. 96) ou «logical process», ou encore "mental process». Par contre, le geste physiographique - c'est-à-dire iconique (objet) ou kinétographique (action) - renvoie à «mental object» (Efron, 1972: 97). La différence cognitive entre gestes physiographiques et idéographiques est ce à quoi renvoie le geste : objet ou processus.

Par ailleurs, un rôle non négligeable, nous allons le voir, est imparti aux déictiques ou gestes de pointage, qui consistent à indiquer quelque chose par une direction du geste dans l'espace. Nous n'y inclurons pas le pointage discursif (l'index orienté vers le haut, par exemple) dont la direction n'est pas ciblée. La direction peut être indiquée par l'index ou le pouce. La main ouverte peut aussi indiquer une direction (comme, par exemple, le geste qui désigne poliment l'interlocuteur en disant vous), mais cela n'a pas été considéré ici comme geste déictique, car ce geste est difficile à distinguer d'un geste de la même forme à caractère discursif. La cible du geste déictique peut être présente in situ (geste dit "déictique concret» ou de "définition ostensive») ou représentée de façon simulée (geste "déictique abstrait»). Les gestes déictiques s'appliquent aussi à la temporalité dans le discours (par exemple, hier ou avant est souvent un geste DERRIÈRE ${ }^{5}$; voir Calbris, 1985). Le pointage pour énumération est aussi inclus dans le geste déictique.

\section{Ellipse et gestes déictiques}

17 Tout d'abord, signalons que les sujets non japonais montrent une certaine similitude comportementale avec le LN. D'après nos observations, le sujet qui a le plus de points communs avec LN est RE, sujet d'origine espagnole (niveau B). Par exemple, RE utilise souvent une gesticulation bi-manuelle tant en symétrie pour signifier sans verbal, par exemple, c'est tout, voilà qu'en mouvement faisant alterner une main puis l'autre (on trouve bizarre gesticulé de la sorte dans le corpus). Tout cela suggère que les cultures gestuelles française et espagnole sont proches, probablement de par la proximité géographique. Cependant, cette hypothèse géographique de la culture gestuelle est contradictoire avec le fait que VA, sujet argentin (niveau C), ressemble à $\mathrm{RE}$, avec qui VA partage seulement une langue commune (l'espagnol). VA utilise aussi relativement beaucoup de gestes bi-manuels. 

symbolique. Ils utilisent un certain nombre de gestes similaires, notamment métaphoriques et idéographiques, que n'emploient pas les sujets japonais. Par exemple, un geste métaphorique classique pour exprimer un peu ou un petit peu, ne s'observe pas, pour rendre cette signification, chez les sujets japonais à l'intérieur de notre corpus. Ce geste rapproche le pouce de l'index, comme pour marquer une épaisseur ou une petite quantité. Bien que tous les sujets comprennent ce geste sans difficulté, le taux et le contexte d'emploi paraissent différents entre sujets japonais et non japonais.
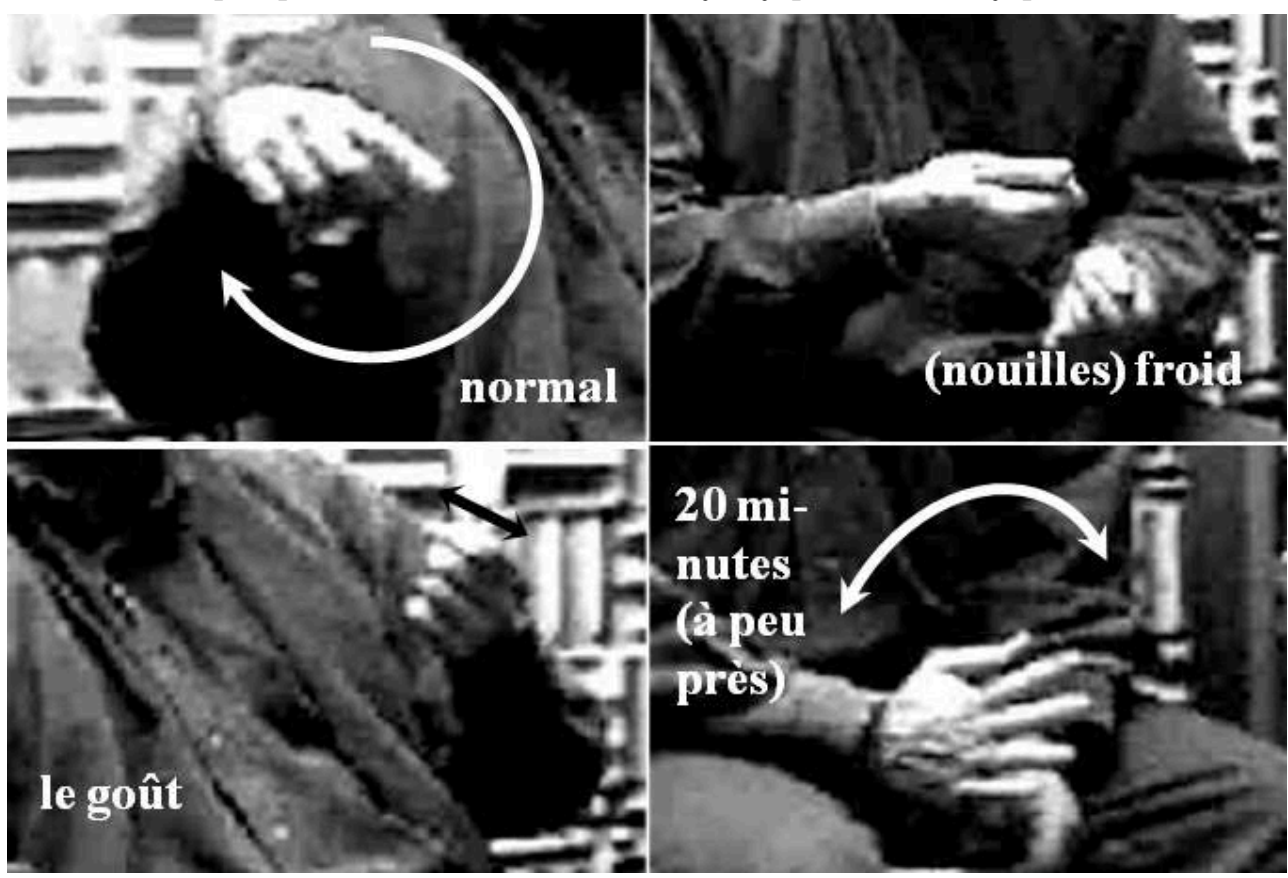

19

Notons qu'il y a bien d'autres gestes métaphoriques du LN ou des LNNs non japonais. Ici, en sont relevés quatre (voir les illustrations ci-contre) : le pointage circulaire renvoyant à l'expression normal ; le geste bi-manuel de saisie exprimant le qualificatif froid ; le geste des doigts frottés les uns contre les autres (utilisé, le plus souvent, pour ARGENT) exprimant ici le goût chez RE ; le geste de l'approximation, à peu près. Les deux derniers gestes sont attestés dans le comportement de LN et YO, sujet japonais (niveau B).

20 Voici, dans l'exemple 1, un premier exemple de geste déictique. La locutrice argentine [VA] est en conversation avec un partenaire d'origine japonaise (SI). Ce dernier connaît la différence de prononciation entre les œufs et un œuf, VA ne connait pas ce mot.
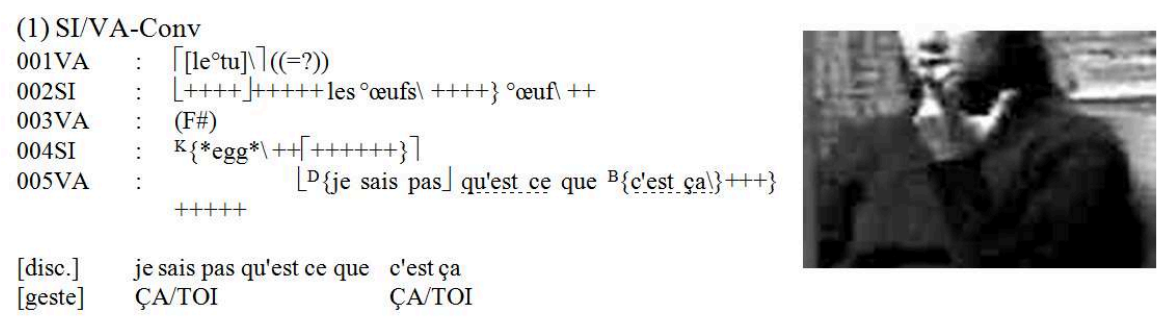

Ici, le geste sert de glose méta-énonciative, devant l'intervention corrective de SI, VA réagit, un peu agacée. Son geste impliquant son interlocuteur en témoigne, voir le sens 
attribué au geste (ÇA/TOI), ce dernier n'ayant aucune référence verbale. Le geste prend en charge un commentaire qui correspond à une ellipse. Plus précisément, le pointage de VA s'oriente à la fois vers l'interlocuteur qu'est-ce que tu dis? et vers l'objet dans son discours voilà ce que moi je dis. Une telle gesticulation est fréquente chez VA, mais est peu observée dans le comportement des sujets japonais, sauf AK et $\mathrm{Mr}^{6}$ pour qui le geste déictique implique l'objet ou l'événement à désigner, ou bien la direction du procès verbal. Le pointage de VA s'accompagne d'un énoncé interrogatif, mais cela peut se trouver avec un énoncé constatif. Il en est ainsi dans l'exemple 2 de RE où AS, japonaise lui explique la recette des sobas :

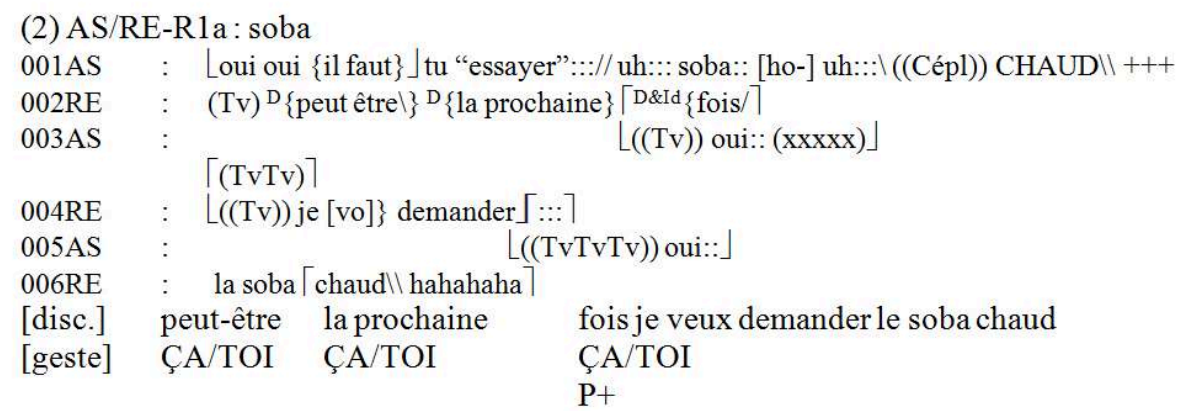

Dans la schématisation qui suit, est présentée une mise en relation du verbal, du discursif et du geste. Retenons que ce qui correspond à l'ellipse est placée entre parenthèses (en gras, ci-dessous) ${ }^{7}$ :
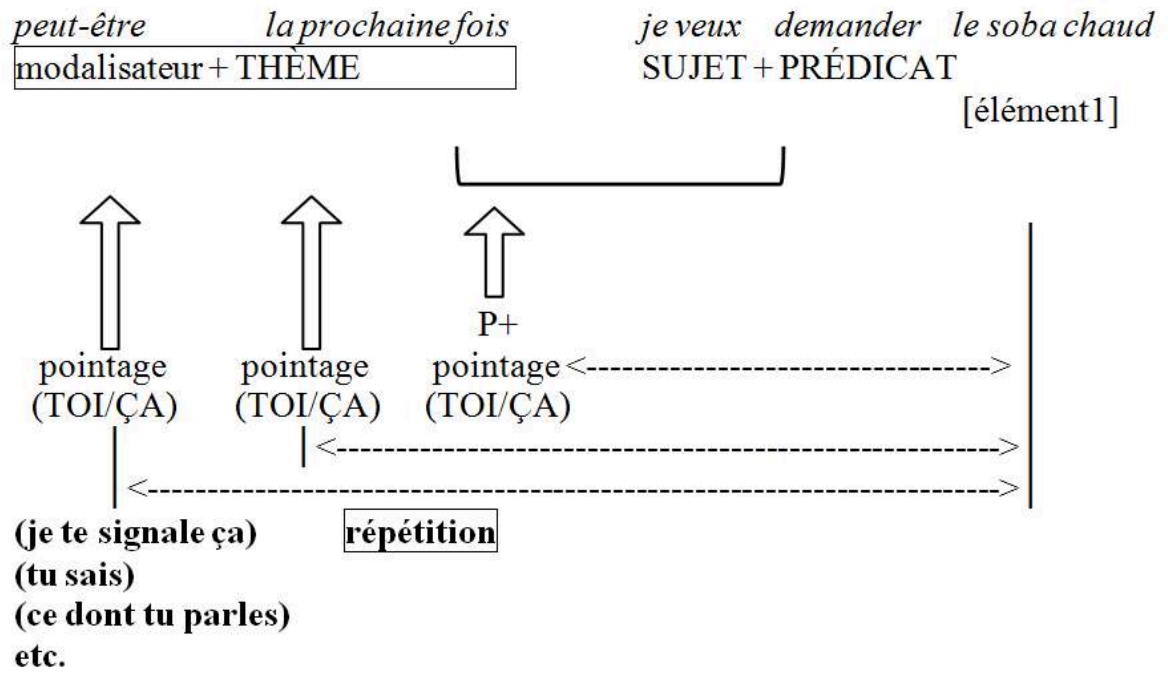

Le geste déictique de VA et RE peut être interprété comme ce que tu dis ou ça dans ton énoncé. C'est là que nous localisons l'ellipse. Voici quelques énoncés avec lesquels VA l'utilise : c'est un plat japonais, non?; c'est pas typique japonais?; tu aimes faire ça?; c'est ça? Le geste déictique lié à l'interlocuteur existe aussi dans la culture gestuelle au Japon. Seulement, il apparaît dans une situation particulière où le gesticulateur désigne très directement l'interlocuteur (souvent avec une interjection marquée) : en fait, il équivaut à une deixis concrète ou une définition ostentatoire comme toi ou ça. Par contre, le geste déictique de VA s'intègre dans le discours, comme une glose insérée. Cela se voit aussi clairement dans le geste déictique de RE. 

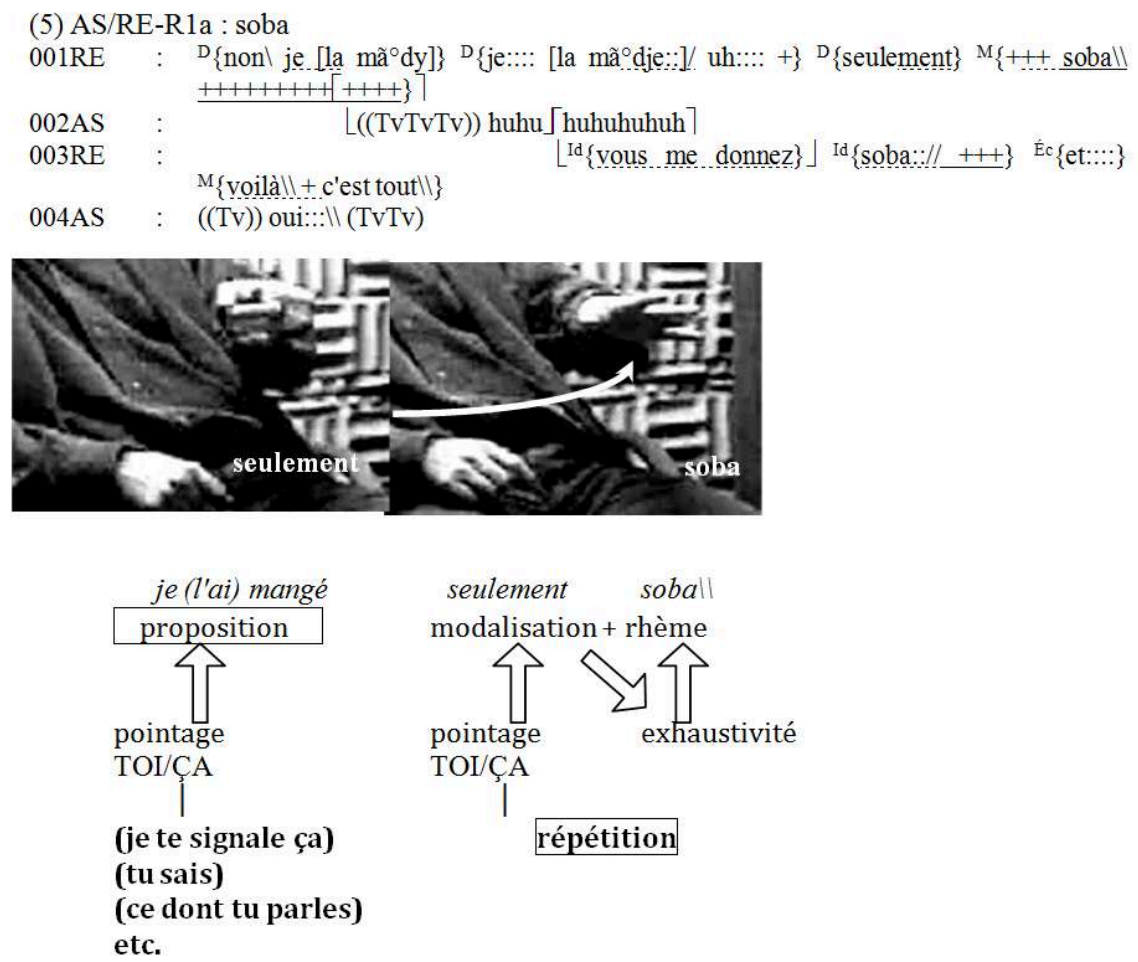

Préférant pratiquer une ellipse du verbal tu sais ou je te signale, RE utilise le geste déictique, qui s'oppose à un autre geste (métaphorique). Le contraste formel et kinésique que créent ces gestes caractérise le discours en deux parties, car ils ont différents centres énonciatifs: un, interactif, autour de la conversation avec son partenaire, l'autre, descriptif, autour de son expérience au Japon. La partie interactionnelle correspond en fait au performatif de l'énoncé et la partie descriptive, au constatif. L'emploi du geste déictique comme performatif n'est pas observé aussi fréquemment chez LN et n'est pas attesté chez les sujets japonais et les autres non japonais dans le cadre de notre corpus. 


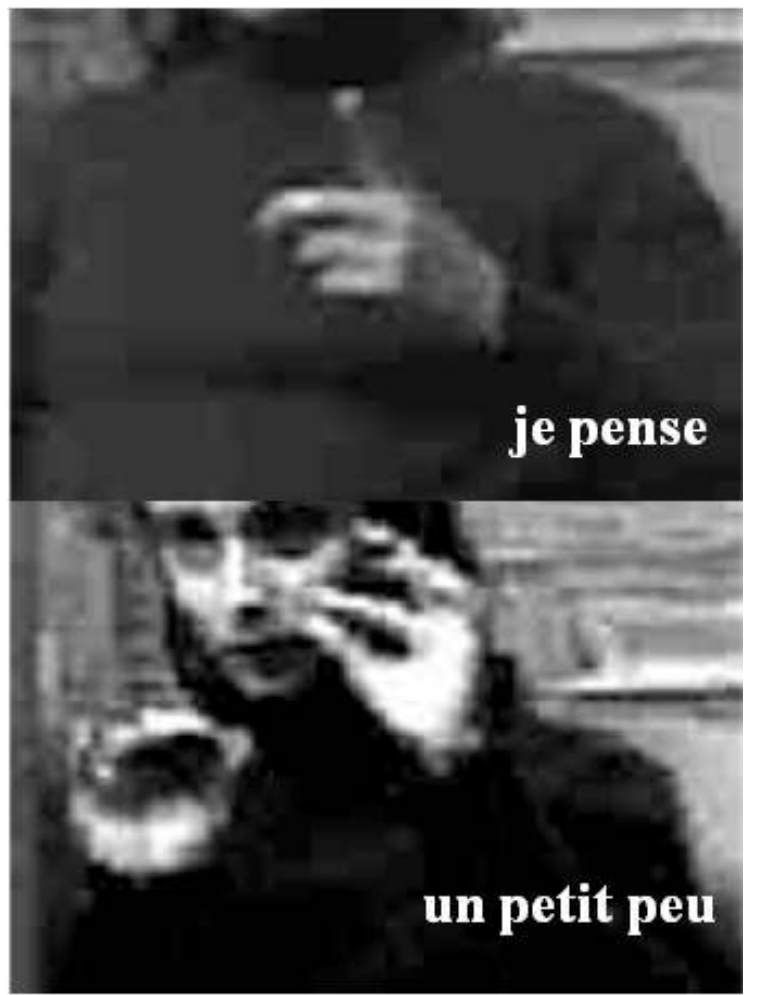

Quant au pointage discursif pouvant correspondre à la glose métadiscursive c'est important, il est observé chez tous les sujets. VA, RE et le LN l'utilisent, néanmoins, avec des expressions variées, comme je pense, renvoyant à l'énoncé performatif. Ainsi, le pointage des sujets non japonais et du LN a trait à la subjectivité ou l'intersubjectivité dans le discours plutôt qu'à une simple traduction visuelle de l'action ou de l'objet. Ici, on peut supposer que le comportement gestuel peut être influencé par la dimension pragmatique de la langue espagnole plutôt que la proximité géographique - entre France et Espagne -, pour le partage d'une culture gestuelle. VA (hispanophone d'Argentine) ressemble plus à RE (hispanophone d'Espagne) qu'au LN (francophone de France), malgré certaines similitudes avec ce dernier.

\section{Ellipse et gestes idéographiques}

Une autre spécificité chez les sujets non japonais est l'emploi des gestes idéographiques. 

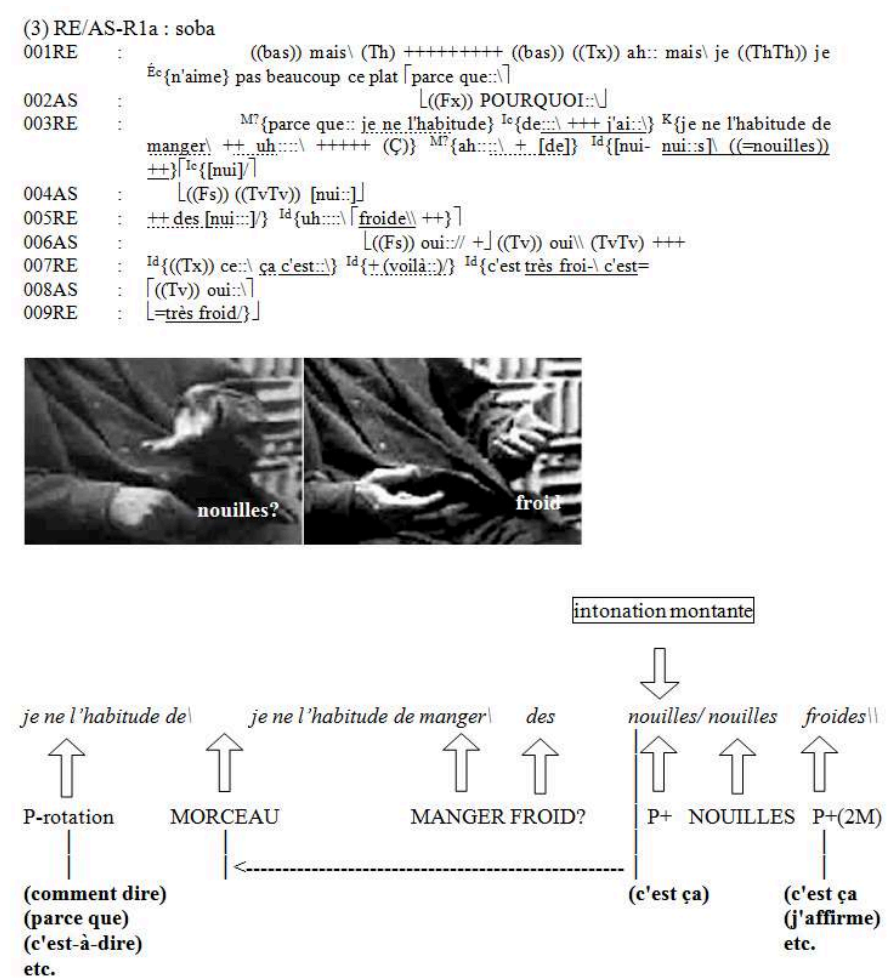

Dans cet extrait, RE marque gestuellement chaque expression clé. Le premier geste est une rotation du poing fermé, probablement associé à l'expression avoir l'habitude. RE abandonne l'énoncé avec un geste iconographique MORCEAU, qui anticipe le contenu du message suivant, et reprend l'énoncé. Ce geste désigne quelque chose de consistant, un morceau, de la nourriture et, par extension des nouilles. Ici, le verbe (manger), le substantif (nouilles) et le qualificatif (froid) sont respectivement gesticulés. Seulement pour l'expression nouilles, RE effectue une demande de confirmation auprès de AS avec un geste idéographique et un geste iconographique exagéré. Pour froid, en dernière position de l'énoncé (005-007), RE finit par un geste idéographique (exprimant sans doute un sentiment d'abandon). Chacun des gestes idéographiques de RE sous-tend l'aspect illocutoire, tout en encadrant les gestes physiographiques, en collaboration avec l'intonation.

La deuxième partie n'a que les gestes idéographiques ( $\mathrm{P}+$ ).
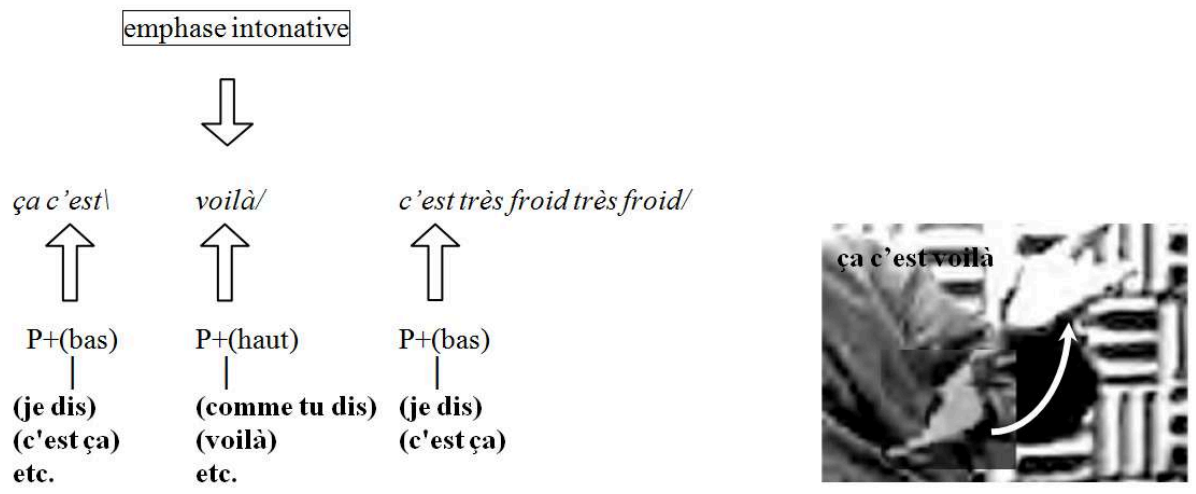

gestuel que verbal. Le deuxième présentatif impliquant l'interlocuteur accompagne l'expression voilà, comme si le geste disait comme tu dis. Les gestes idéographiques de RE sous-tendent, de la sorte, la dimension pragmatique de l'énoncé.

(43) AS/RE-R1a : 318 (soba)

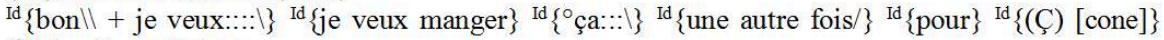

Id $\{$ bien $[$ le gut $] \backslash\}++$

emphase intonative

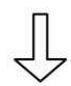

jeveux \ je veux manger ça

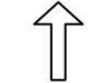

$\mathrm{P}+$ (haut)

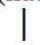

(comme tu dis)(je dis)

(ce que tu dis) (ça)

(voilà) etc.

etc.

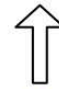

$\mathrm{P}+($ rotation $)$

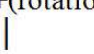

etc.

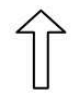

$\mathrm{P}+($ bas $)$

|

(comme tu dis) (ce que tu dis) (voilà) une autre fois/ pour connaître bien le goût \\

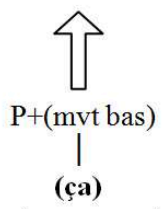

(comme ça)

etc.

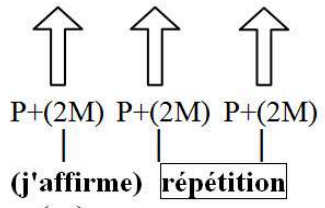

(ca)

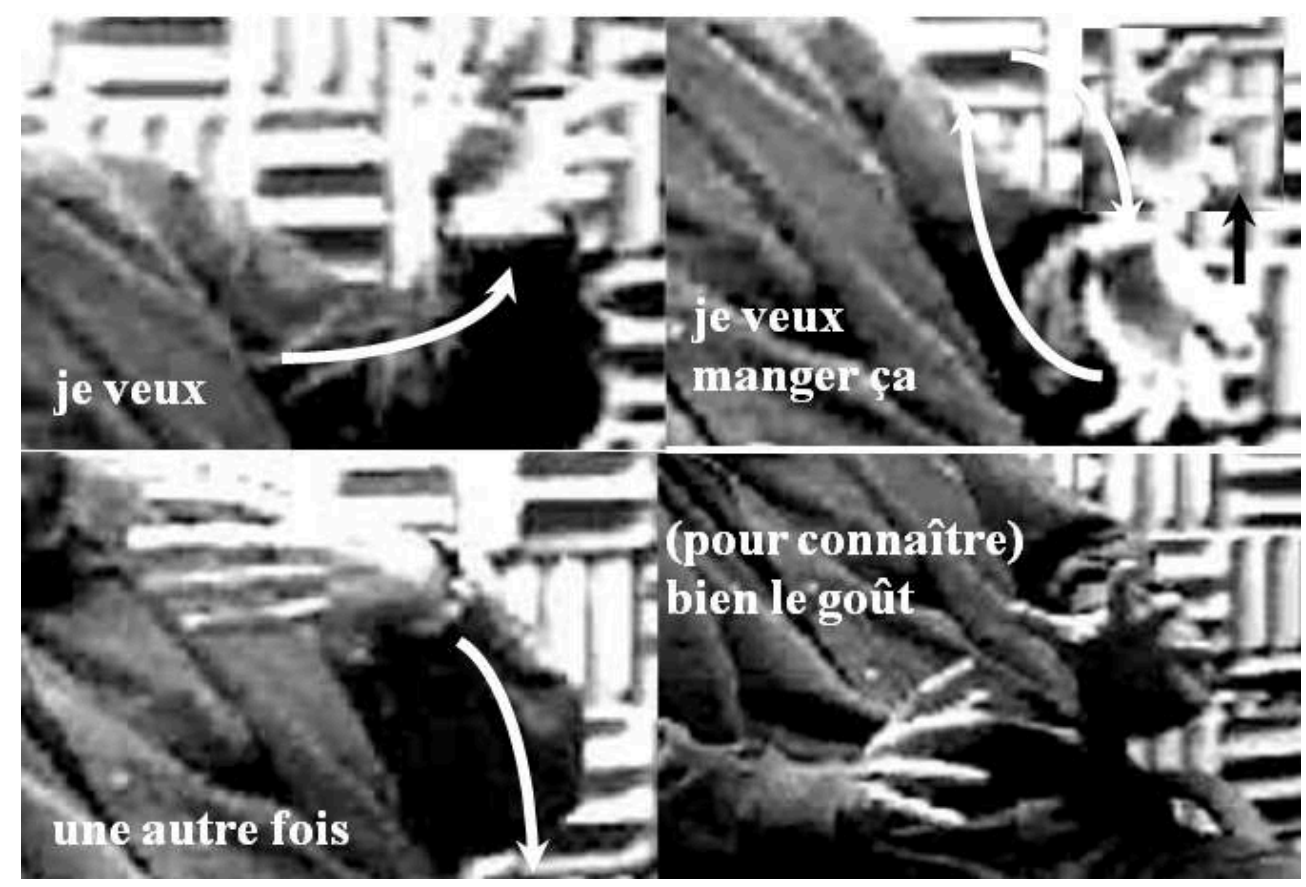

30 Les gestes idéographiques que multiplie RE ne sont pas identiques : le premier (associé à je veux), un mouvement vers l'interlocuteur, est comme déclaration, le second (je veux manger) et le quatrième (une autre fois), comme affirmation, le troisième (ça), pour souligner ce dont parle RE, et enfin le quatrième « resserre » le dernier message (pour connaitre bien le goût) pour conclure son énoncé. Chaque geste suggère un acte illocutoire de l'énoncé. Ainsi, RE ponctue le discours avec ces gestes en explorant la différence formelle et kinésique, ainsi que le positionnement des mains dans l'espace de 
gesticulation. Quelques-uns des gestes de RE sont proches des gestes métaphoriques. Subtilité formelle ou kinésique, la gestion spatiale de l'activité gestuelle chez RE, VA et LN dépasse les possibilités de notre système de codage.

31 L'habitus gestuel de VA et RE consiste à marquer gestuellement chacune des parties du discours. Si le LN et quelques sujets non japonais (RE et VA) partagent un certain nombre de traits formels et kinésiques, la variation du rapport chronologique entre geste et discours n'est pas notable chez eux : l'utilisation du critère chronologique est moins complexe. RE et VA font, en effet, usage de l'anticipation ou du retard gestuel, mais la plupart des gesticulations tendent à être redondantes ou complétives, et n'engendrent pas d'effet précis sur le discours.

On peut se demander si cette spécificité comportementale vient de leur propre culture gestuelle (la thèse de transfert) ou résulte d'une appropriation gestuelle (la thèse d'acculturation), mais il sera difficile de répondre à cette question faute de données pertinentes. La comparaison avec les autres sujets non japonais laisse entrevoir, néanmoins, que la thèse de transfert serait la plus plausible. TD, sujet danois (niveau C), est en effet assez différent de VA et RE sur les aspects tant formel que kinésique ${ }^{8}$. Bien que son comportement implique des gestes métaphoriques, ils sont souvent ambigus; les gesticulations circulaires, bi-manuelles et/ou symétriques sont beaucoup moins fréquemment employées que par VA, RE et LN. Or, leur niveau de compétence de discours ou la durée d'exposition à la culture cible sont comparables (sauf RE, qui, lecteur d'espagnol à l'université, est mieux exposé à la communauté d'accueil).

Cependant, les sujets non japonais linguistiquement moins compétents sont gestuellement plus proches de la plupart des sujets japonais à niveau égal de compétence. Par rapport à HA, sujet américain (niveau D), un autre sujet américain qui n'a pas fait l'objet de cette étude, montre quelques similitudes avec VA et RE quant à l'emploi des gestes idéographiques et métaphoriques. Il y a donc tout de même appropriation gestuelle chez les sujets américains. Il sera alors prudent de considérer que transfert de la culture source et appropriation gestuelle coexistent dans le comportement des sujets non natifs.

\section{Observations conclusives}

Dans ce cadre de l'interaction exolingue, la compréhension mutuelle des partenaires de l'échange reste la préoccupation majeure et nous avons vu à l'œuvre les modalités verbale, non verbale et vocale. Pour ces deux dernières modalités, dans le cas d'une ellipse verbale, les éléments principaux jouant, respectivement, un rôle positif dans cette compréhension sont les gestes physiographiques ou métaphoriques et l'emphase. Entre autres, le geste peut matérialiser une relation syntaxique mal exprimée ou implicite.

Le jeu du raccourci explicatif, c'est-à-dire de la focalisation prosodique et visuelle est certainement plus complexe en face à face qu'en situation de privation visuelle. Le corpus Kida (2005) en témoigne, l'ellipse peut être de deux sortes. La première due à un déficit lexical du LNN correspond à un évitement, la seconde peut faire l'objet d'une négociation dans le but d'une intercompréhension. Toujours est-il que toutes deux constituent, sans aucun doute, des moments précieux pour la compréhension de 
l'interaction. Debaisieux et al. (2008), prenant le contrepied de Culicover et Jackendoff (2005), l'expriment ainsi :

L'unité communicative apparemment «ellipsée» est en fait une unité communicative «noyau » non verbale et paraverbale, c'est-à-dire portée soit par une conduite communicative 'en production' du locuteur soit par une conduite communicative 'en interprétation' de l'interlocuteur.

Certes, l'ellipse peut donc être une procédure d'évitement, un principe de précaution lorsqu'un natif interagit avec un non-natif, mais elle peut aussi correspondre, comme en témoignent les sections 4 et 5 , à un marquage pragmatique de l'interaction: glose méta-énonciative, identification des centres énonciatifs (constatif vs performatif)... Pour préciser, l'absence de verbal ou la partie ellipsée peut être volontaire et donc stratégique, c'est alors le non verbal qui endosse ce qui n'est pas énoncé dans le discours en L2.

\section{BIBLIOGRAPHIE}

Barthes, Rolland (1970). L'empire des signes. Genève, A. Skira.

Calbris, Geneviève (1985). « Espace-temps : expression gestuelle du temps », Semiotica, 55 (1/2) : 43-73.

Cosnier, Jacques (2012). « Axiomes de la communication multimodale », in Robert Vion, Alain Giacomi \& Claude Vargas (éds.), La corporalité du langage. Aix-en-Provence, Publications de l'Université de Provence : 103-106.

Creider, Chet A. (1978). « Intonational tone groups and body motion in Luo conversation ", Anthropological linguistics, 20 : 327-339.

Creider, Chet A. (1986). « Interlanguage comparaisons in the study of the interactional use of gesture ", Semiotica, 62 (1/2) (Special issue : Approaches to gesture, édité par Adam Kendon \& Thomas D. Blakely) : 147-164.

Culicover, Peter W. \& Jackendoff, Ray, (2005) Simpler Syntax. Oxford University Press.

De Jorio, Andrea (1832). La mimica degli antichi investigata nel gestire napoletano. Napoli, Della stampiera e cartiera del fibreno.

Debaisieux, Jeanne-Marie, Deulofeu, José \& Martin, Philippe (2008). « Pour une syntaxe sans ellipse ", in Jean-Christophe Pitavy \& Michèle Bigot (dir.), Ellipse et effacement : du schème de phrase aux règles discursives. Publications de l'Université de Saint-Etienne : 227- 235.

Eco, Umberto (1983). Postille al nome della rosa (aggiunte all'ed. italiana tascabile di Il nome della rosa, Milano : Bompiani ; Apostille au nom de la rose. Grasset, pour la traduction française, 1985).

Efron, David (1972). Gesture, race and culture : A tentative study of some of the spatio- temporal and "linguistic" aspects of the gestural behavior of Eastern Jews and Southern Italians in New York City, living under similar as well as different environmental condi- tions. The Hague, Mouton (1ère édition en 1941). 
http://www.noslangues-ourlanguages.gc.ca/bien-well/fra-eng/style/ellipse-fra.html (consulté le 15 juin 2013).

Engel Johann Jacob (1785-86). Ideen zu einen Mimik. Berlin, Auf Kosten des Verfassers und in Commission bey August Mylius (tr. fr. : Idées sur le geste et l'action théâtrale. Paris, H.J. Jansen et Comp, 1795 ; reproduction originale de l'édition française : Genève, Slatkine Reprints, 1979).

Ferguson, Charles A. (1971). «Absence of copula and the notion of simplicity : A study of normal speech, baby talk, foreigner talk and pidgins », in Dell Hymes (ed.), Pidginization and creolization of languages. New York, Cambridge University Press : 141-150.

Kellerman, Eric \& Van Hoof, Anne-Marie (2003). « Manual accents », IRAL, 41 (3) : 251-269.

Kida, Tsuyoshi (2003). « Le rôle des indices visuels dans la compréhension discursive en langue seconde ", Marges Linguistiques : 260-285.

Kida, Tsuyoshi (2005). Appropriation du geste par les étrangers : le cas de japonais apprenant le français. Thèse de doctorat, Université Aix-Marseille 1.

Kida, Tsuyoshi (2008). " Does gesture aid discourse compréhension in the L2 », in Steve McCafferty \& Gale Stam (eds.), Gesture. Second language acquisition and classroom research. London/ New York, Routledge : 131-156.

Kida, Tsuyoshi (à paraître). Geste et appropriation. Acculturation non verbale chez des étrangers sous l'effet de la mondialisation. Publications de l'Université de Provence.

Kida, Tsuyoshi \& Faraco, Martine (2003). « Gestures in second language discourse :

Metacommunicative function and perlocution », in Monica Rector, Isabella Poggi \& Nadine Trigo (eds.), Gestures : Meaning and use. Porto, Universidad Fernando Pessoa : 305-318.

Kida, Tsuyoshi \& Faraco, Martine (2009). « La prédication gestuelle », Faits de Langues, 30-31 : 217-226.

Kida, Tsuyoshi \& Faraco, Martine (sous presse). « Gestuelle de l'aspect ou aspect de la gestuelle? « Aspectualisateur » d'une interlangue du français », Faits de langues.

Kita, Sotaro (1993). Language and thought interface: A study of spontaneous gestures and Japanese mimetics. Ph.D. Thesis, University of Chicago, Department of Psychology, Department of Linguistics.

Larsen-Freeman, Diane \& Long, Michael (1991). An introduction to second language acquisition research. London, Longman.

Long, Michael H. (1996). « The role of the linguistic environment in second language acquisition ", in William C. Ritchie \& Tej K. Bhatia (eds.), Handbook of second language acquisition. San Diego, Academic Press : 413-468.

Müller, Cornelia (1994). « Semantic structure of motional gestures and lexicalization patterns in Spanish and German descriptions of motion-events », CLS (Chicago Linguistic Society), 30 (1) : 281-295.

Portail linguistique du Canada, par Le Bureau de la traduction, organisme de service spécial de Travaux publics et Services gouvernementaux Canada

Stam, Gale (1998). « Changes in patters of thinking about motion with L2 acquisition ", in Serge Santi, Isabelle Guaïtella, Christian Cavé \& Gabrielle Konopczynski (éds.), Oralité et gestualité : communication multimodale, interaction. Paris, L'Harmattan : 615-619.

Stam, Gale (2006). « Thinking for speaking about motion : L1 and L2 speech and gesture ", IRAL, $44(2): 145-171$. 
Talmy, Leonard (1991). « Path to realization : A typology of event conflation », Berkeley Working Papers in Linguistics : 480-519.

Talmy, Leonard (2000). Toward a cognitive semantics. Volume 1 : Concept structuring systems. Volume 2 : Typology and process in concept structuring. Cambridge, MA : MIT Press.

Trésor de la Langue Française Informatisé. http://atilf.atilf.fr (consulté le 15 juin 2013)

Van Hoof, Anne-Marie \& Kellerman, Eric (2001). « Gesture placement in motion events : A crosslinguistic study of L1 and L2 'thinking for speaking' patterns ", Paper Presented at the 11th conference of European Second Language Association (Eurosla), 26th-29th September 2001, University of Paderborn (Germany).

Yoshioka, Keiko \& Kellerman, Eric (2006). « Gestural introduction of Ground reference in L2 narrative discourse », IRAL, 44 (2), 171-193.

\section{ANNEXES}

\section{Conventions}

Conventions pour le texte

MAJUSCULES : geste interprété

italiques : extrait d'exemple ou expression soulignée

« termes » : citation ou terme sujet à discussion

"termes" : termes mis entre guillemets dans la citation

Conventions de transcription du corpus

Yaa $\rceil \leq b b\rfloor$ : début/fin de chevauchement

$\{a\}$ : début/fin de gesticulation

k, d, b, Id, M, Éc, Ic : Indique la typologie à laquelle appartient le geste qui suit. Dans nos exemples : kinétographique, déictique, battement, idéographique, métaphorique, échec, iconique.

+ : pause $(«+»=0,1 \mathrm{sec})$

$::$ allongement vocalique (durée approximative $::=0,1 \mathrm{sec}$ )

// : intonation montante discontinue

/ : intonation montante continue

$\backslash$ : intonation descendante continue ou discontinuité neutre

Mot : accent non attendu jamais réalisé devant flèche

(mot) : mot inaudible inféré (« $x »$, syllabe non inférée)

$>$ aaa $<$ : débit rapide

a : a :a : : débit ralenti

$\mathrm{p}(\mathrm{h})$ eut (h)être : rires en cours d'élocution

((bas)) : commentaire du transcripteur

Tv/Th/Tx : mouvement vertical/horizontal/particulier de la tête (voir NB) 


\section{Fs : sourire (voir NB)}

(Cépl) : mouvement du corps, épaules.

NB. Quelques signes (ex. Tv) sont signalés soit entre (()), soit entre (), la première notation indiquant que le mouvement est concomitant à la production verbale, la seconde que ce mouvement s'insère exactement à cet endroit de la transcription.

\section{NOTES}

1. 間 Le ma désigne l'intervalle, l'espace, la durée, la distance, non celle qui sépare, mais celle qui unit. Son kanji représente un soleil (petit kanji au milieu) entouré par une porte. Le 間合い (maai) renvoie, pour les arts martiaux, à l'espace entre deux adversaires, espace qui détermine le rituel du salut et de l'engagement du combat; non seulement l'écart entre les deux adversaires, mais aussi le temps qu'il faut pour le franchir. Le Kanji 合pour "ai" représente un pot recouvert d'un couvercle et symbolise deux choses qui s'accordent ensemble, l'union, l'harmonie.

2. Pour les données complètes de l'enregistrement, voir Kida (2005), volume 2 (corpus).

3. Niveau (A) Avancé : AK, AY, Niveau (B) Intermédiaire fort: YO, Mr1, SA [RE] Niveau (C) Intermédiaire faible : Mr2, YS, HI [TD] [VA] Niveau (D) Elémentaire : MS, AS, SI, SZ [HA]. Les noms entre crochets carrés ([]) indiquent un participant non natif non japonais. Parmi les 12 participants japonais apparaissent donc deux avancés, trois respectivement pour les deux niveaux intermédiaires et quatre pour les débutants.

4. Efron utilise aussi les termes «logico-topographique» et «logico-pictorial» comme synonymes d'idéographiques. Il mentionne aussi que sa notion d'idéographique ou de logicopictorial correspond à ce que Cicéron (Oratoire, Livre III) appelle «significatio ", et à ce qu'Engel (1785-86/1795) appelle « analogique ».

5. Dans notre transcription, les majuscules renvoient au sens attribué au geste, par le transcripteur analyste.

6. Voir note 2.

7. Il en est ainsi dans tous les exemples qui suivent.

8. La culture gestuelle de l'Europe du nord est globalement différente de celle de l'Europe du sud. Néanmoins, il convient de rester prudent car il y a des différences à l'intérieur de chaque bloc, comme l'a noté de Jorio (1832) pour l'Italie du sud. Ainsi il existe des gestes typiquement italiens qu'on ne trouve pas dans le sud-est de la France, pourtant proche de l'Italie à bien des niveaux.

\section{RÉSUMÉS}

Ce travail sur la compréhension orale en langue seconde (L2) dans une interaction exolingue s'appuie sur le «totexte» ou énoncé total, défini par Cosnier (2012: 104) comme l'ensemble formé du texte (verbal) et du cotexte. En effet, dans une interaction en face à face, la structure du discours oral est jalonnée par un certain nombre d'indices gestuels et prosodiques aussi bien en situation de communication endolingue qu'exolingue (Kida, à paraître ; Kida \& Faraco, 2009). Or, la mise en emphase (accent gestuel) obéit à des contraintes propres à chaque langue (voir, entre autres, les travaux de Müller, 1994, Kita, 1993, Stam, 2006), ce qui conduit les locuteurs à utiliser une sorte de syntaxe gestuelle régie par leur langue dominante. C'est ainsi que ce type $\mathrm{d}^{\prime}$ ' accent 
gestuel » (Creider, 1978, 1986 ; Kida \& Faraco, 2009) ou « manual accent » (Kellerman \& van Hoof, 2003) est souvent transféré d'une langue à l'autre, lors de l'apprentissage d'une langue seconde (Stam, 1998 ; van Hoof \& Kellerman 2001 ; Yoshioka \& Kellerman, 2006).

À partir de ces constats, a été conduite une étude sur les gestes produits par des apprenants en langue seconde (désormais L2) puisque le transfert du comportement d'accent manuel de la langue maternelle à la L2 prouve l'existence d'une grammaticalité de la modalité gestuelle dans le discours. Nous avons d'ores et déjà travaillé (Kida et Faraco, à paraitre) sur l'appropriation de la mise en gestes de japonophones apprenant le français comme L2, donc pour deux langues ayant une 'grammaticalité' gestuelle comparable, tout au moins pour ce qui est de l'accent manuel. En effet, japonais et français sont tous deux classés dans le groupe des langues à cadrage verbal et non dans celui des langues à cadrage satellite (Talmy, 1991 et 2000, entre autres).

Cette contribution veut préciser les observations précédentes portant sur l'appropriation de l'oral, du prosodique et du gestuel d'une langue cible (le français) par des locuteurs non natifs (LNNs), Après quelques remarques générales sur la façon dont se comportent les modalités gestuelle et vocale en cas d'absence du verbal dans le discours explicatif d'un locuteur natif (LN), tentant de se faire comprendre de son partenaire non natif, nous verrons comment la focalisation non verbale fonctionne au plan pragmatique en cas d'ellipse verbale chez le LNN.

Le corpus de référence (Kida, 2005) propose des séquences vidéo de locuteurs natifs (LNs) et non natifs s'expliquant mutuellement des recettes de cuisine de leur propre pays. Deux conditions d'enregistrement ont été mises en place : l'une où les interlocuteurs se voient et l'autre où les interlocuteurs ne se voient pas, car séparés par un écran opaque. Les LNNs sont pour la plupart des japonophones (de niveau linguistique variable) mais également des locuteurs d'autres langues maternelles (danois, espagnol, anglais). Les enregistrements ont eu lieu dans une chambre anechoïque de l'université, les participants ont interagi en dyade, sur le schéma LN-LNN ou LNN-LNN , et nous nous intéresserons ici, plus particulièrement, aux échanges contenant des exemples d'ellipse syntaxique (fait caractéristique de l'oral) : alors qu'il manque d'information verbale, la question qui se pose est de savoir s'il y a continuité prosodique et quel rôle y joue l'activité gestuelle.

Après avoir défini l'ellipse comme un " raccourci explicatif », les auteurs soulignent l'ambiguïté de ce procédé car il peut tout aussi bien faciliter l'intercompréhension que la brouiller. Toujours est-il que le nombre des ellipses du LN augmente quand les partenaires non natifs sont de niveaux linguistiques moins avancés, mais que la condition de visibilité/non-visibilité n'offre pas de grand contraste; en interaction avec un LNN de niveau avancé, par contre, l'ellipse du LN augmente en situation d'écran par rapport à la situation de face à face. Globalement, l'ellipse peut être une stratégie d'interaction du LN pour que le discours soit plus aisé et rapide à suivre par son partenaire en interaction exolingue. Soulignons toutefois que l'utilisation de l'ellipse est non seulement liée au niveau de compétence en L2 de l'interlocuteur non natif, mais aussi à la disponibilité de l'information visuelle qui accompagne le discours.

Lors de ces observations dans ce contexte elliptique, nous avons vu que la compréhension discursive repose surtout sur des gestes tels qu'idéographiques ou déictiques. Pour ces gestes, le comportement gestuel des LNNs non japonophones se rapproche de celui du LN. Reste à savoir si cela est fonction de la proximité géographique de la langue-culture d'appartenance ou de la proximité fonctionnelle de celle-ci.

Certes, nous l'avons dit, l'ellipse peut être une procédure d'évitement, un principe de précaution lorsqu'un natif interagit avec un non-natif, mais elle peut aussi correspondre, comme en témoignent certains échanges dans le corpus de référence, à un marquage pragmatique de l'interaction, en l'absence de verbal: glose méta-énonciative, identification des centres énonciatifs (constatif $v s$ performatif)... Pour préciser, l'absence de verbal ou la partie ellipsée peut être volontaire et donc stratégique, c'est alors le non verbal qui endosse ce qui n'est pas énoncé dans le discours en $\mathrm{L} 2$. 
This work focusing on listening comprehension in second language (L2) in an exolingual interaction is based on the "totext" - or total utterance - defined by Cosnier (2012: 104) as a combination of the text (verbal) and cotext. In face-to-face interaction the speech structure is indeed marked out with several gestural and prosodic cues in both endolingual and exolingual communication (Kida, to be published; Kida \& Faraco, 2009). Yet, the emphasis (gestural accent) follows constraints specific to each language (see, among others, Müller, 1994; Kita, 1993; Stam, 2006) which forces the speakers to use a sort of gestural syntax governed by their dominant language. This is how this type of "accent gestuel" (Creider, 1978, 1986; Kida \& Faraco, 2009) or "manual accent" (Kellerman \& van Hoof, 2003) is often transferred from one language to the other during second-language acquisition (Stam, 1998; van Hoof \& Kellerman, 2001; Yoshioka \& Kellerman, 2006).

Based on these observations, a study on second language learners' gestures has been conducted since the transfer of manual accent behavior from the mother tongue to the L2 proves the existence of a grammaticality of gestural modality in speech. Work has already been done (Kida \& Faraco, to be published) on the appropriation of gestures by Japanese speakers learning French as a second language -that is to say for two languages that have a similar gestural "grammaticality", at least in regards to manual accent. Japanese and French are indeed classified in the group of verb-framed languages and not in the satellite-framed one (Talmy, 1991 and 2000, among others).

This paper intends to specify the observations previously made on oral, prosodic and gestural appropriation of a target language (French) by non-native speakers (NNS). After some general comments on the way the gestural and vocal modalities behave when the verbal is absent from the explanatory discourse of a native speaker trying to be understood by his non-native partner, we will study how the non-verbal focusing works on the pragmatic level when verbal ellipsis is used by the NNS.

The reference corpus (Kida, 2005) proposes video footage of native and non-native speakers mutually explaining recipes from their own country. Two recording conditions were put in place: one where the interlocutors see each other and one where they do not because they are separated by an opaque screen. The NNS are for the most part Japanese speakers (of various linguistic levels) but are also speakers of other native languages (Danish, Spanish, English). Recordings took place at the university in an anechoic chamber, participants interacted in dyad, following the NS-NNS or NNS-NNS pattern and this work will focus more precisely on exchange including example of syntactic ellipsis (a distinctive feature of oral communication): when verbal information is missing, the point is to know whether there is a prosodic continuity and what part the gestural activity plays in it.

After having defined the ellipsis as an "explanatory shortcut", the authors underline the ambiguity of this process because it can make the intercomprehension easier as well as it can blur it. Nonetheless, the number of ellipsis made by the NS increases when the non-native speakers are linguistically less advanced but when the visibility/non visibility feature does not show much difference; however, when interacting with an advanced NNS, the NS's ellipsis increase when the screen is on compared to the face-to-face interaction. Overall, in an exolingual interaction ellipsis can be a strategy of interaction used by the NS to make the speech easier and faster to follow for his partner. However, it should be added that the use of ellipsis is not only linked to the non-native speaker's L2 level of proficiency but also to the availability of the visual information that comes with speech.

During these observations made in this elliptical context we saw that the discursive comprehension mostly rests upon gestures - ideographic or deictic. For these gestures, the non Japanese-speaking NNS's gestural behavior gets close to the NS's one. It remains to be seen whether this is due to the geographic proximity of the language-culture they belong to or to its functional proximity. 
As we have said before, when a native interacts with a non-native ellipsis can be an avoidance procedure, a precautionary principle, but when the verbal is missing it can also act -as some of the exchange in the reference corpus reveal- as a pragmatic marking of the interaction: metaenunciative gloss, identification of the enunciative centers (constative vs performative)... To clarify, the absence of the verbal or the ellipsised part can be intentional and thus strategic; it is then the non-verbal that assumes what is not stated by speech in L2.

\section{INDEX}

Keywords : native/non native interaction, gesture, listening comprehension, second language, ellipsis, illocution

Mots-clés : interaction natif/non-natif, geste, compréhension orale, langue seconde, ellipse, illocutoire

\section{AUTEURS}

\section{TSUYOSHI KIDA}

University de Tsukuba, Japon

kida.tsuyoshi.ga@u.tsukuba.ac.jp

\section{MARTINE FARACO}

Aix-Marseille Université, CNRS, LPL UMR 7309, Aix-en-Provence, France

Martine.faraco@lpl-aix.fr 